\title{
ANALYTICAL SOLUTION FOR MULTILAYER PLATES USING GENERAL LAYERWISE PLATE THEORY
}

\author{
$U D C 624.011 .1: 624.073 .8(045)=20$
}

\author{
Đorđe Vuksanović, Marina Ćetković \\ University of Belgrade, Faculty of Civil Engineering, Belgrade, Serbia and Montenegro
}

\begin{abstract}
This paper deals with closed-form solution for static analysis of simply supported composite plate, based on generalized laminate plate theory (GLPT). The mathematical model assumes piece-wise linear variation of in-plane displacement components and a constant transverse displacement through the thickness. It also include discrete transverse shear effect into the assumed displacement field, thus providing accurate prediction of transverse shear stresses. Namely, transverse stresses satisfy Hook's law, 3D equilibrium equations and traction free boundary conditions. With assumed displacement field, linear strain-displacement relation, and constitutive equations of the lamina, equilibrium equations are derived using principle of virtual displacements. Navier-type closed form solution of GLPT, is derived for simply supported plate, made of orthotropic laminae, loaded by harmonic and uniform distribution of transverse pressure. Results are compared with $3 D$ elasticity solutions and excellent agreement is found.
\end{abstract}

\section{INTRODUCTION}

In the last decades, scientists have made considerable progress in understanding behavior of composite laminates. It is noticed that anisotropic multilayered structures posses transverse discontinuous mechanical properties and higher transverse shear and transverse normal stress deformability. In order to model such material behavior, two different approaches have arise, that is equivalent single-layer theories (ESL) and layerwise theories (LWT).

In single-layer theories one single expression is used through entire thickness to explain the displacement field of the plate. By this, deformation of multilayer plate is described by equivalent single layer, thus reducing $3 \mathrm{D}$ problem to $2 \mathrm{D}$ problem. In order to include transverse shear deformation, classical (CLPT) and shear deformation theories have been developed. Namely, CLPT based on Kirchhoff's hypothesis, ignores the effect of transverse shear deformation. On the other hand, FSDT based on Raissner and 
Mindlin, assume constant transverse shear stresses in the thickness direction, giving a need for shear correction factors to adjust for unrealistic variation of the shear strain/stress. In order to overcome the limitations of CLPT and FSDT, Higher-order Shear Deformation Theories (HSDT) which involve higher-order terms in Taylor's expansion of the displacements in the thickness coordinate were developed.
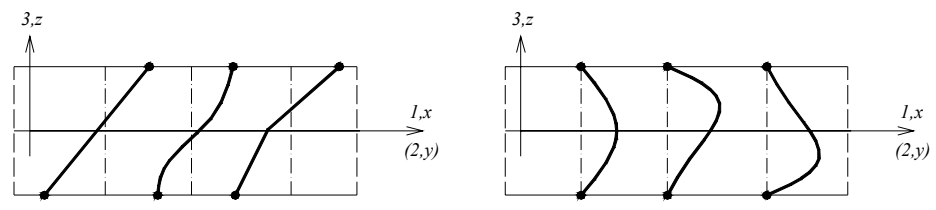

ISOTROPIC - SINGLE LAYER PLATE
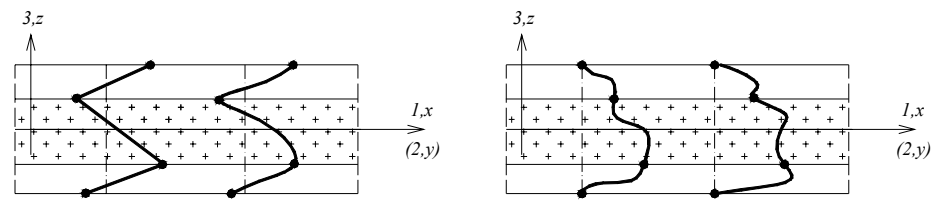

ANISOTROPIC - MULTILAYER PLATE

Fig. 1. Displacement and shear stress distribution across the plate thickness for isotropic and anisotropic plates

In wish of obtaining the accurate prediction of stress distribution and precisely model kinematics of laminated composites, three-dimensional state of stresses have to be analyzed. Despite conventional 3D elasticity theory, new family of layerwise theories (LWT) have been developed. Naimely, in LWT displacement field is defined for each layer, thus including discrete material and discrete shear effects into the assumed displacement field. Also, it is noticed that LWT models have some analyze advantages over the conventional 3D models. First, as LWT model allows independent in-plane and through the thickness interpolation, the element stiffness matrix can be computed much faster. Second, even the volume of input data is reduced, LWT are capable of achieving the same level of solution accuracy as a conventional 3D models.

This paper deals with displacement based on layerwise theory of Reddy (1987), so called Generalized Laminated Plate Theory (GLPT). The theory is based on piece-wise linear variation of in-plane displacement components and constant transverse displacement through the thickness. The Navier-type closed form solution is presented for simply supported plate loaded by harmonic and uniform distribution of transverse pressure. The main objective is to compare results of the mentioned theory to $2 \mathbf{D}$ and $\mathbf{3 D}$ theories, and to develop mathematical model that will be more efficient than conventional 3D model. 


\section{GeneraLized LAYERWISE Plate THEORY}

\subsection{Assumptions}

The following assumptions are used in the analysis of plate model:

1. Material follows Hooke's Law and each layer is made of orthotropic material.

2. Strain-displacement relation is linear, i.e. geometrical linearity.

3. Displacement and stress distributions over the $\mathrm{z}$ thickness plate direction is determined by Lagrangian linear interpolation functions.

4. The inextensibility of normal is imposed.

\subsection{Displacement field}
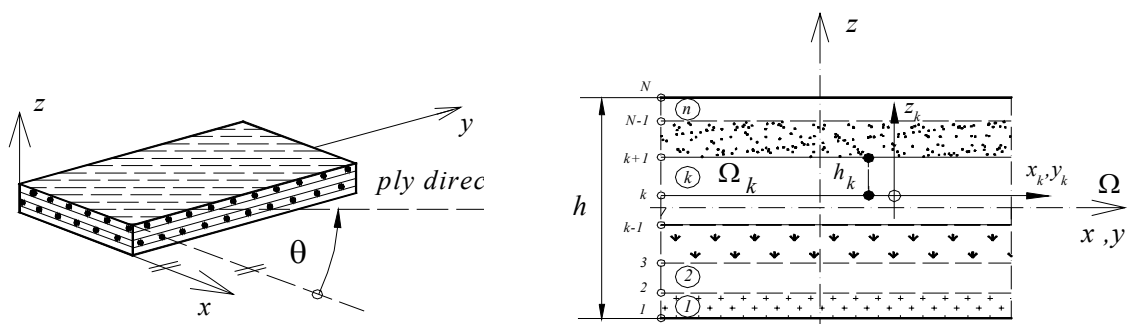

Fig. 2. Multilayer composite plate

Consider a laminated plate (Fig. 2) composed of $n$ orthotropic laminae. The integer $k$, denotes the layer number that starts from the plate bottom. Plate middle surface coordinates are $(x, y, z)$, while layer coordinates are $\left(x_{k}, y_{k}, z_{k}\right)$. Plate and layer thickness are denoted as $h$ and $h_{k}$, respectively.

The displacements components $\left(u_{1}, u_{2}, u_{3}\right)$ at a point $(x, y, z)$ can be written as:

$$
\begin{aligned}
& u_{1}(x, y, z)=u(x, y)+U(x, y, z) \\
& u_{2}(x, y, z)=v(x, y)+V(x, y, z) \\
& u_{3}(x, y, z)=w(x, y)
\end{aligned}
$$

where $(u, v, w)$ are the displacements of a point $(x, y, 0)$ on the reference plane of the laminate, and $U, V$ are functions which vanish on the reference plane:

$$
U(x, y, 0)=V(x, y, 0)=0
$$

Let now reduce $3-\mathrm{D}$ model to $2 \mathrm{D}$ format, by the following approximations:

$$
\begin{aligned}
& U(x, y, z)=\sum_{I=1}^{N} U^{I}(x, y) \cdot \Phi^{I}(z) \\
& V(x, y, z)=\sum_{I=1}^{N} V^{I}(x, y) \cdot \Phi^{I}(z)
\end{aligned}
$$

where $U^{I}$ and $V^{I}$ are undetermined coefficients, and $\Phi^{I}(z)$ are layerwise continuous functions of the thickness coordinate. In the view of finite element approximation, the functions $\Phi^{I}(z)$ are the one-dimensional (linear, quadratic or cubic) Lagrange interpolation 
functions of the thickness coordinates (Fig. 3), and $\left(U^{I}, V^{I}\right)$ are the values of $\left(u_{1}, u_{2}\right)$ at the I-th plane.
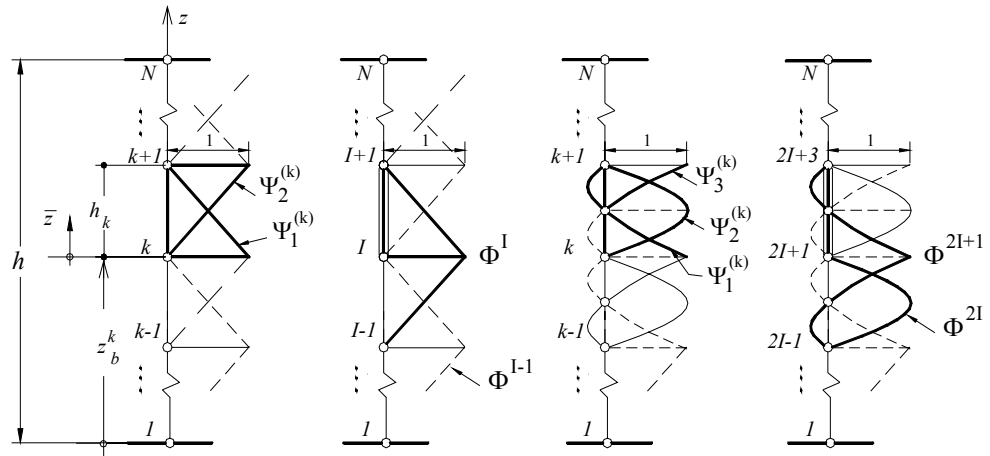

Fig. 3. Local and global linear and quadratic Lagrangian interpolation functions

If we assume linear interpolation of in-plane displacement components through the thickness, linear Lagrangian interpolation functions will have the form:

$$
\begin{aligned}
& \Phi^{1}(z)=\Psi_{1}^{(1)}(z) \quad z_{1} \leq z \leq z_{2} \\
& \Phi^{I}(z)=\left\{\begin{array}{lc}
\Psi_{2}^{(I-1)}(z) & z_{I-1} \leq z \leq z_{I} \\
\Psi_{1}^{(I)}(z) & z_{I} \leq z \leq z_{I+1}
\end{array} \quad(I=2,3, \ldots, N-1)\right. \\
& \Phi^{N}(z)=\Psi_{1}^{(N)}(z) \quad z_{N-1} \leq z \leq z_{N} \\
& \text { where: } \quad \Psi_{1}^{(k)}=1-\frac{\bar{z}}{h_{k}} \quad \Psi_{2}^{(k)}=\frac{\bar{z}}{h_{k}} \quad 0 \leq \bar{z} \leq h_{k}
\end{aligned}
$$

Since the displacement field of GLPT is represented as linear combination of product of functions of in-plane coordinates and functions of thickness coordinates, independent in-plane and through the thickness discretization of the plate may be achieved. Also, as the thickness variation of displacement components is defined in terms of piecewise Lagrangian interpolation functions, the in-plane displacement components will be continuous through the laminate thickness.

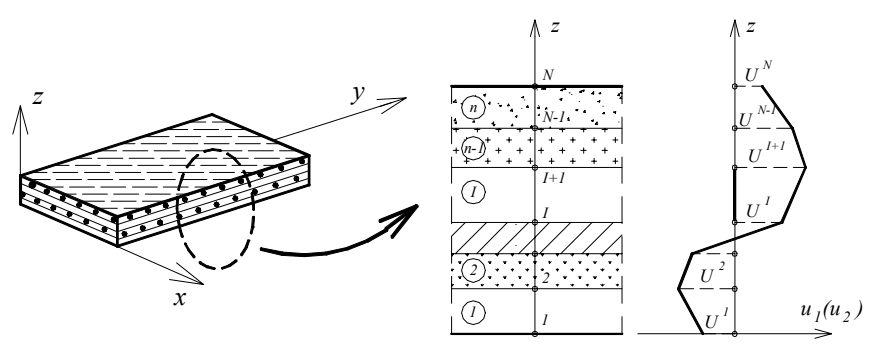

Fig. 4. Displacement field through the laminate thickness 


\subsection{Strain-displacement relations of the laminae}

The linear strain-displacement relations are given as:

$$
\begin{aligned}
& \varepsilon_{x x}=\frac{\partial u}{\partial x}+\sum_{I=1}^{N} \frac{\partial U^{I}}{\partial x} \Phi^{I} \\
& \varepsilon_{y y}=\frac{\partial v}{\partial y}+\sum_{I=1}^{N} \frac{\partial V^{I}}{\partial y} \Phi^{I} \\
& \gamma_{x y}=\frac{\partial u}{\partial y}+\frac{\partial v}{\partial x}+\sum_{I=1}^{N}\left(\frac{\partial U^{I}}{\partial y}+\frac{\partial V^{I}}{\partial x}\right) \Phi^{I} \\
& \gamma_{x z}=\sum_{I=1}^{N} U^{I} \frac{d \Phi^{I}}{d z} \\
& \gamma_{y z}=\sum_{I=1}^{N} V^{I} \frac{d \Phi^{I}}{d z}
\end{aligned}
$$

From the assumed displacement and deformation field we conclude that in-plane deformation components $\left(\varepsilon_{x x}, \varepsilon_{y y}, \gamma_{x y}\right)$ will be continuous through the plate thickness. while the transverse strains $\left(\gamma_{x z}, \gamma_{y z}\right)$ need not to be continuous.

\subsection{Constitutive equations of laminae}

Laminated plate is made of laminae having a fibers oriented at an angle $\theta$, measured from the material $\bar{x}$ to global $x$ axis (Fig. 5). The stress strain relations of the laminae is therefore defined in material coordinate system as:

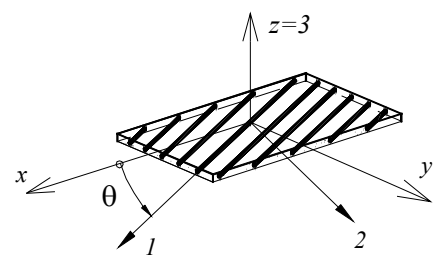

Fig. 5. Global and local coordinate system of laminae

$$
\left\{\begin{array}{l}
\sigma_{1} \\
\sigma_{2} \\
\tau_{12} \\
\tau_{13} \\
\tau_{23}
\end{array}\right\}^{(k)}=\left[\begin{array}{ccccc}
C_{11} & C_{12} & 0 & 0 & 0 \\
C_{12} & C_{22} & 0 & 0 & 0 \\
0 & 0 & C_{33} & 0 & 0 \\
0 & 0 & 0 & C_{44} & 0 \\
0 & 0 & 0 & 0 & C_{55}
\end{array}\right\}^{(k)}\left\{\begin{array}{c}
\varepsilon_{1} \\
\varepsilon_{2} \\
\gamma_{12} \\
\gamma_{13} \\
\gamma_{23}
\end{array}\right\}
$$

where:

$$
\begin{aligned}
& \boldsymbol{\sigma}^{(k)}=\left\{\begin{array}{lllll}
\sigma_{1} & \sigma_{2} & \tau_{12} & \tau_{13} & \tau_{23}
\end{array}\right\}^{(k)^{T}} \text { stress components of } k \text {-th laminae } \\
& \text { in material coordinates } \\
& \boldsymbol{\varepsilon}^{(k)}=\left\{\begin{array}{lllll}
\varepsilon_{1} & \varepsilon_{2} & \gamma_{12} & \gamma_{13} & \gamma_{23}
\end{array}\right\}^{(k)^{T}} \quad \text { strain components of } k \text {-th laminae } \\
& \text { in material coordinates } \\
& \mathbf{C}_{i j}^{(k)} \text { matrix of material elastic coefficients for } k \text {-th laminae, given as: }
\end{aligned}
$$




$$
\begin{aligned}
& \Delta=1-v_{12} v_{21}-v_{23} v_{32}-v_{31} v_{13}-2 v_{13} v_{21} v_{32}, \\
& C_{11}=\frac{E_{1}\left(1-v_{23} v_{32}\right)}{\Delta}, \quad C_{22}=\frac{E_{2} 1-v_{31} v_{13}}{\Delta}, \quad C_{12}=C_{21}=\frac{E_{2}\left(v_{21}+v_{31} v_{23}\right)}{\Delta}, \\
& C_{33}=G_{12}, \quad C_{44}=G_{13}, \quad C_{55}=G_{23} .
\end{aligned}
$$

where:

$E_{1}, E_{2}, E_{3} \quad$ Young's moduli in 1,2 and 3 directions, respectively

$v_{i j} \quad$ Poisson's ratio-defined as ratio of transverse strain in $j$-direction to axial strain in $i$-direction $(i, j=1,2,3)$

$G_{12}, G_{23}, G_{13}$ shear moduli in the 1-2, 2-3 and 1-3 planes, respectively

Along with following relations:

$$
\frac{v_{i j}}{E_{i}}=\frac{v_{j i}}{E_{j}} \quad(i, j=1,2,3)
$$

Since all quantities should be referred to a single coordinate system, we need to establish transformation relations among stresses and strains in global system to the corresponding quantities in material (local) coordinate system. The constitutive matrix in global coordinate system will than be of the form:

$$
\mathbf{Q}_{i j}{ }^{(k)}=\mathbf{T}^{-1} \mathbf{C}_{i j}{ }^{(k)} \mathbf{T}
$$

where:

$$
\mathbf{T}=\left[\begin{array}{ccccc}
\cos ^{2} \theta & \sin ^{2} \theta & 2 \sin \theta \cos \theta & 0 & 0 \\
\sin ^{2} \theta & \cos ^{2} \theta & -2 \sin \theta \cos \theta & 0 & 0 \\
-\sin \theta \cos \theta & \sin \theta \cos \theta & \cos ^{2} \theta-\sin ^{2} \theta & 0 & 0 \\
0 & 0 & 0 & \cos \theta & \sin \theta \\
0 & 0 & 0 & -\sin \theta & \cos \theta
\end{array}\right]
$$

The Hooke's law for the $k$-th laminae in the global coordinate system is now:

$$
\left\{\begin{array}{c}
\sigma_{x x} \\
\sigma_{y y} \\
\tau_{x y} \\
\tau_{x z} \\
\tau_{y z}
\end{array}\right\}^{(k)}=\left[\begin{array}{ccccc}
Q_{11} & Q_{12} & Q_{13} & 0 & 0 \\
Q_{12} & Q_{22} & Q_{23} & 0 & 0 \\
Q_{13} & Q_{23} & Q_{33} & 0 & 0 \\
0 & 0 & 0 & Q_{44} & Q_{45} \\
0 & 0 & 0 & Q_{45} & Q_{55}
\end{array}\right]^{(k)} \times\left\{\begin{array}{l}
\varepsilon_{x x} \\
\varepsilon_{y y} \\
\gamma_{x y} \\
\gamma_{x z} \\
\gamma_{y z}
\end{array}\right\}^{(k)}
$$

where:

$\boldsymbol{\sigma}^{(k)}=\left\{\begin{array}{lllll}\sigma_{x x} & \sigma_{y y} & \tau_{x y} & \tau_{x z} & \tau_{y z}\end{array}\right\}^{(k)^{T}}$ stress components of $k$-th laminae in global coordinates $\boldsymbol{\varepsilon}^{(k)}=\left\{\begin{array}{lllll}\varepsilon_{x x} & \varepsilon_{y y} & \gamma_{x y} & \gamma_{x z} & \gamma_{y z}\end{array}\right\}^{(k)^{T}}$ strain components of $k$-th laminae in global coordinates 


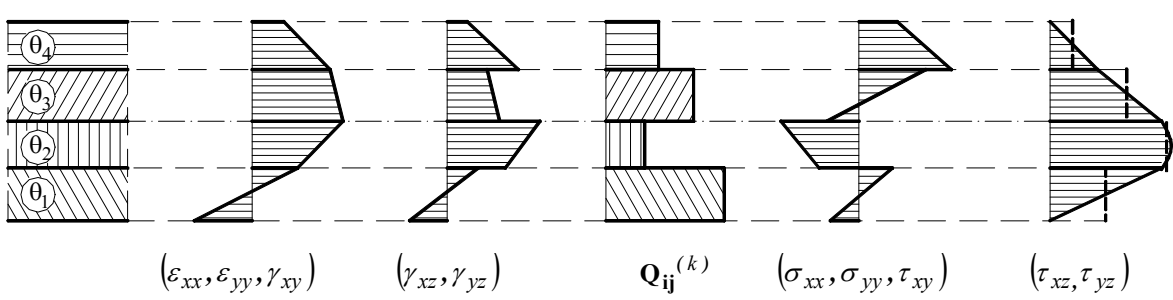

Fig. 6. Assumed deformation and stress field in laminated composite plate

From equation (9) and already mentioned distribution of strain field through the plate thickness, we conclude (Fig. 6) that in-plane stresses will be discontinuous at dissimilar material layers, leaving the possibility for transverse stresses to be continuous through the plate thickness. This transverse stresses are the one that satisfy constitutive relations, 3D equilibrium equations and traction free boundary conditions.

\subsection{Equilibrium equations}

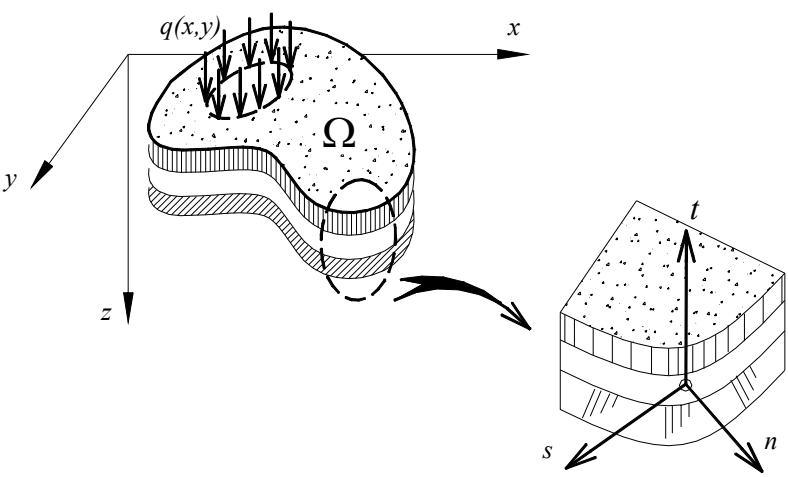

Fig. 7. Geometry and load of a plate with curved boundary

The equilibrium equations are derived using the principle of virtual displacements:

$$
0=\delta U+\delta V
$$

where $\delta U$ is virtual strain energy, $\delta V$ is virtual work done by applied forces, which are given as:

$$
\begin{aligned}
\delta U= & \int_{\Omega}\left\{\int_{-h / 2}^{h / 2}\left[\sigma_{x x} \delta \varepsilon_{x x}+\sigma_{y y} \delta \varepsilon_{y y}+\tau_{x y} \delta \gamma_{x y}+\tau_{x z} \delta \gamma_{x z}+\tau_{y z} \delta \gamma_{y z}\right] d z\right\} d x d y \\
\delta V= & -\int_{\Omega}\left[q_{b}\left(x, y,-\frac{h}{2}\right) \delta w_{0}\left(x, y,-\frac{h}{2}\right)+q_{t}\left(x, y, \frac{h}{2}\right) \delta w_{0}\left(x, y, \frac{h}{2}\right)\right] d x d y- \\
& \oint_{\Gamma}\left\{\int_{-h / 2}^{h / 2}\left[\hat{\sigma}_{n n} \delta u_{n}+\hat{\sigma}_{n s} \delta u_{n s}+\hat{\sigma}_{n t} \delta w\right] d z\right\} d s
\end{aligned}
$$


In the following text, we assume that distributed load acts in the middle surface and the stress resultants are given as:

$$
\begin{aligned}
& \left\{\begin{array}{l}
N_{x x} \\
N_{y y} \\
N_{x y}
\end{array}\right\}=\int_{-h / 2}^{h / 2}\left\{\begin{array}{l}
\sigma_{x x} \\
\sigma_{y y} \\
\tau_{x y}
\end{array}\right\} d z,\left\{\begin{array}{l}
N^{I} x \\
N^{I} y \\
N^{I} x
\end{array}\right\}=\int_{-h / 2}^{h / 2}\left\{\begin{array}{c}
\sigma_{x x} \\
\sigma_{y y} \\
\tau_{x y}
\end{array}\right\} \Phi^{I} d z, \\
& \left\{\begin{array}{l}
Q_{x} \\
Q_{y}
\end{array}\right\}=\int_{-h / 2}^{h / 2}\left\{\begin{array}{l}
\sigma_{x z} \\
\sigma_{y z}
\end{array}\right\} d z,\left\{\begin{array}{l}
Q^{I}{ }_{x} \\
Q^{I} y
\end{array}\right\}=\int_{-h / 2}^{h / 2}\left\{\begin{array}{l}
\sigma_{x z} \\
\sigma_{y z}
\end{array}\right\} \frac{d \Phi^{I}}{d z} d z, \\
& \left\{\begin{array}{l}
\hat{N}_{n n} \\
\hat{N}_{n s} \\
\hat{Q}_{n}
\end{array}\right\}=\int_{-h / 2}^{h / 2}\left\{\begin{array}{l}
\hat{\sigma}_{n n} \\
\hat{\sigma}_{n s} \\
\hat{\sigma}_{n t}
\end{array}\right\} d z .
\end{aligned}
$$

If we substitute (13) into (12),(11) we will get the following $2 N+3$ partial differential equations in $2 N+3$ variables $\left(u, v, w, U^{I}, V^{I}\right),(I=1, \ldots N)$ :

$$
\begin{aligned}
& \delta u=0: \quad N_{x x, x}+N_{x y, y}=0 \\
& \delta v=0: \quad N_{x y, x}+N_{y y, y}=0 \\
& \delta w=0: \quad Q_{x, x}+Q_{y, y}+q=0 \\
& \begin{array}{lll}
\delta U^{I}=0: & N^{I}{ }_{x x, x}+N^{I}{ }_{x y, y}-Q^{I}{ }_{x}=0 \\
\delta V^{I}=0: & N^{I}{ }_{x y, x}+N^{I}{ }_{y y,}-Q^{I}=0
\end{array} \quad I=1, \ldots N
\end{aligned}
$$

with appropriate geometric boundary conditions:

$$
\left(u, v, w, U^{I}, V^{I}\right)
$$

and force boundary conditions:

$$
N_{n n}+\sum_{I=1}^{N} N^{I}{ }_{n n}-\hat{N}_{n n}=0, N_{n s}+\sum_{I=1}^{N} N^{I}{ }_{n s}-\hat{N}_{n s}=0, Q_{n}+\sum_{I=1}^{N} Q^{I}{ }_{n}-\hat{Q}_{n}=0
$$

where:

$$
\begin{array}{ll}
N_{n n}=N_{x x} n_{x}+N_{x y} n_{y} & N^{I} n n=N^{I} x x n_{x}+N^{I} x y n_{y} \\
N_{n s}=N_{x y} n_{x}+N_{y y} n_{y} & N^{I} n s=N^{I} x y n_{x}+N^{I} y y n_{y} \\
Q_{n}=Q_{x} n_{x}+Q_{y} n_{y} & Q^{I}{ }_{n}=Q^{I} x n_{x}+Q^{I} y n_{y}
\end{array}
$$




\subsection{Constitutive equations of laminate}

The constitutive equations of laminate are given as:

$$
\begin{aligned}
& \left\{\mathbf{N}^{0}\right\}=[\mathbf{A}]\left\{\boldsymbol{\varepsilon}^{0}\right\}+\sum_{I=1}^{N}\left[\mathbf{B}^{\mathrm{I}}\right]\left\{\boldsymbol{\varepsilon}^{\mathrm{I}}\right\} \\
& \left\{\mathbf{N}^{\mathrm{I}}\right\}=\left[\mathbf{B}^{\mathrm{I}}\right]\left\{\boldsymbol{\varepsilon}^{0}\right\}+\sum_{J=1}^{N}\left[\mathbf{D}^{\mathrm{JI}}\right]\left\{\boldsymbol{\varepsilon}^{\mathrm{J}}\right\}
\end{aligned}
$$

where:

$$
\begin{aligned}
& \left\{\mathbf{N}^{0}\right\}=\left\{N_{x x} \quad N_{y y} \quad N_{x y} \quad Q_{x} \quad Q_{y}\right\}^{T} \text { force components in the middle surface }
\end{aligned}
$$

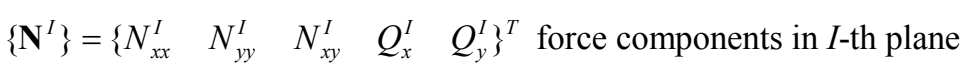

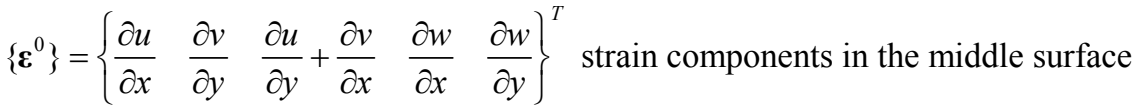

$$
\begin{aligned}
& \left\{\boldsymbol{\varepsilon}^{I}\right\}=\left\{\frac{\partial U^{I}}{\partial x} \quad \frac{\partial V^{I}}{\partial y} \quad \frac{\partial U^{I}}{\partial y}+\frac{\partial V^{I}}{\partial x} \quad U^{I} \quad V^{I}\right\}^{T} \quad \text { strain components in } I \text {-th plane }
\end{aligned}
$$

Constitutive matrixes of the laminate are given as:
$[\mathbf{A}]=\left[A_{p q}\right]=\sum_{k=1}^{n} \int_{z_{k}}^{z_{k+1}}\left[Q_{p q}{ }^{(k)}\right] d z$
$p, q=1,2,3,4,5$
$[\mathbf{B}]=\left[B_{p q}^{I}\right]=\sum_{k=1}^{n} \int_{z_{k}}^{z_{k+1}}\left[Q_{p q}{ }^{(k)}\right] \Phi^{I} d z$,
$p, q=1,2,3$
$[\mathbf{B}]=\left[\bar{B}_{p q}^{I}\right]=\sum_{k=1}^{n} \int_{z_{k}}^{z_{k+1}}\left[Q_{p q}{ }^{(k)}\right] \frac{d \Phi^{I}}{d z} d z$,
$p, q=4,5$
$[\mathbf{D}]=\left[D_{p q}^{J I}\right]=\sum_{k=1}^{n} \int_{z_{k}}^{z_{k+1}}\left[Q_{p q}{ }^{(k)}\right] \Phi^{J} \Phi^{I} d z$,
$p, q=1,2,3$
$[\mathbf{D}]=\left[\bar{D}_{p q}^{J I}\right]=\sum_{k=1}^{n} \int_{z_{k}}^{z_{k+1}}\left[Q_{p q}{ }^{(k)}\right] \frac{d \Phi^{J}}{d z} \frac{d \Phi^{I}}{d z} d z$
$p, q=4,5$ 


\section{NAVIER-TyPe ClOSED FORM SOLUTION}

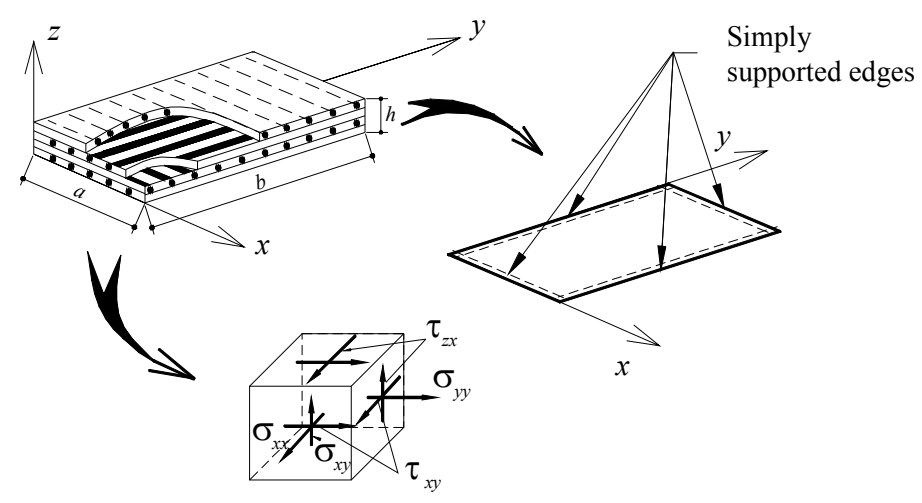

Fig. 8. Geometry and boundary conditions of multilayer plate

For a rectangular ( $a x b$ ) simply supported cross-ply laminated plate (Fig. 8), composed of $n$-layers, the following constitutive coefficients become zero:

$$
A_{13}=A_{23}=A_{45}=B_{13}=B_{23}=\bar{B}_{45}=D_{13}=D_{23}=\bar{D}_{45}=0
$$

The reduced governing equations (14) become:

$$
\begin{aligned}
& A_{11} u_{, x x}+A_{12} v_{, x y}+A_{33}\left(u_{, y y}+v_{, x y}\right)+\sum_{I=1}^{N}\left[B_{11}^{I} U_{, x x}^{I}+B_{12}^{I} V_{, x y}^{I}+B_{33}^{I}\left(U_{, y y}^{I}+V_{, x y}^{I}\right)\right]=0 \\
& A_{12} u_{, x y}+A_{22} v_{, y y}+A_{33}\left(u_{, x y}+v_{, x x}\right)+\sum_{I=1}^{N}\left[B_{12}^{I} U_{, x y}^{I}+B_{22}^{I} V_{, y y}^{I}+B_{33}^{I}\left(U_{, x y}^{I}+V_{, x x}^{I}\right)\right]=0 \\
& A_{44} w_{, x x}+A_{55} w_{, y y}+\sum_{I=1}^{N}\left[B_{44}^{I} U_{, x}^{I}+B_{55}^{I} V_{, y}^{I}\right]+q=0 \\
& \sum_{I=1}^{N}\left[B_{11}^{I} u_{, x x}+B_{12}^{I} v_{, x y}+B_{33}^{I}\left(u_{, y y}+v_{, x y}\right)-B_{44}^{I} w_{, x}\right] \\
& \quad+\sum_{I=1}^{N}\left[D_{11}^{J I} U_{, x x}^{I}+D_{12}^{J I} V_{, x y}^{I}+D_{33}^{J I}\left(U_{, y y}^{I}+V_{, x y}^{I}\right)-\bar{D}_{44}^{J I} U^{I}\right]=0 \\
& \sum_{I=1}^{N}\left[B_{12}^{I} u_{, x y}+B_{22}^{I} v_{, y y}+B_{33}^{I}\left(u_{, x y}+v_{, x x}\right)-\bar{B}_{55}^{I} w_{, x}\right] \\
& \quad+\sum_{I=1}^{N}\left[D_{12}^{J I} U_{, x y}^{I}+D_{22}^{J I} V_{, y y}^{I}+D_{33}^{J I}\left(U_{, x y}^{I}+V_{, y y}^{I}\right)-\bar{D}_{55}^{J I} V^{I}\right]=0
\end{aligned}
$$

The closed form solution that satisfy the boundary conditions

$$
\begin{aligned}
& v=w=V^{I}=N_{x x}=N_{x x}^{I}=0 ; \quad x=0, a ; \quad I=1, \ldots N \\
& u=w=U^{I}=N_{y y}=N_{y y}^{I}=0 ; \quad y=0, b ; \quad I=1, \ldots N
\end{aligned}
$$


and differential equations (18) can be found by assuming the following harmonic form for the unknown variables:

$$
\begin{aligned}
& u=\sum_{m, n}^{\infty} X_{m n} \cos \alpha x \sin \beta x, \quad v=\sum_{m, n}^{\infty} Y_{m n} \sin \alpha x \cos \beta y, \quad w=\sum_{m, n}^{\infty} W_{m n} \sin \alpha x \sin \beta x, \\
& U^{I}=\sum_{m, n}^{\infty} R^{I}{ }_{m n} \cos \alpha x \sin \beta x, \quad V^{I}=\sum_{m, n}^{\infty} S^{I}{ }_{m n} \sin \alpha x \cos \beta y
\end{aligned}
$$

and applied loadings

$$
f(x, y)=\sum_{m, n}^{\infty} q_{m n} \sin \alpha x \sin \beta y
$$

If we substitute $(20)_{1},(20)_{2}$ into the system of $2 N+3$ equations (18) for each of the Fourier modes $(m, n)$, we will get the following algebraic equations in matrix form:

$$
\left[\begin{array}{cc}
{[\mathbf{k}]} & {\left[\mathbf{k}^{I}\right]} \\
{\left[\mathbf{k}^{I}\right]^{\mathbf{T}}} & {\left[\mathbf{k}^{J I}\right]}
\end{array}\right]\left\{\begin{array}{l}
\left\{\boldsymbol{\Delta}_{1}\right\} \\
\left\{\boldsymbol{\Delta}_{2}\right\}
\end{array}\right\}=\left\{\begin{array}{c}
\{\mathbf{f}\} \\
\{\mathbf{0}\}
\end{array}\right\}
$$

for unknowns $\left(X_{m n}, Y_{m n}, W_{m n}, R_{m n}^{I}, S_{m n}^{I}\right)$,

where:

$$
\begin{aligned}
& k_{11}=A_{11} \alpha^{2}+A_{33} \beta^{2}, k_{12}=\left(A_{12}+A_{33}\right) \alpha \beta=k_{21}, k_{22}=A_{22} \beta^{2}+A_{33} \alpha^{2}, \\
& k_{33}=A_{44} \alpha^{2}+A_{55} \beta^{2}, k_{13}=k_{31}=k_{23}=k_{32}=0 ; \\
& k_{11}^{I}=B_{11}^{I} \alpha^{2}+B_{33}^{I} \beta^{2}, k_{12}^{I}=\left(B_{12}^{I}+B_{33}^{I}\right) \alpha \beta=k_{21}^{I}, \\
& k_{22}^{I}=B_{33}^{I} \alpha^{2}+B_{22}^{I} \beta^{2}, k_{31}^{I}=B_{44}^{I} \alpha, k_{32}^{I}=B_{55}^{I} \beta ; \\
& k_{11}^{J I}=D_{11}^{J I} \alpha^{2}+D_{33}^{J I} \beta^{2}+D_{44}^{J I}, k_{12}^{J I}=\left(D_{12}^{J I}+D_{33}^{J I}\right) \alpha \beta=k_{21}^{J I}, k_{22}^{J I}=D_{33}^{J I} \alpha^{2}+D_{22}^{J I} \beta^{2}+D_{55}^{J I} \\
& \Delta_{1}=\left\{\begin{array}{lll}
X_{m n} & Y_{m n} & W_{m n}
\end{array}\right\}^{T}, \Delta_{2}=\left\{R_{m n}^{I} \quad S_{m n}^{I}\right\}^{T},\{f\}=\left\{\begin{array}{lll}
0 & 0 & -q_{m n}
\end{array}\right\}^{T} .
\end{aligned}
$$

Once the coefficients $\left(X_{m n}, Y_{m n}, W_{m n}, R_{m n}^{j}, S_{m n}^{j}\right)$ are obtained, the in-plane stresses are computed from the constitutive equations (6) as:

$$
\left\{\begin{array}{c}
\sigma_{x x} \\
\sigma_{y y} \\
\tau_{x y}^{(k)}
\end{array}\right\}=\left[\begin{array}{ccc}
Q_{11} & Q_{12} & 0 \\
Q_{12} & Q_{22} & 0 \\
0 & 0 & Q_{33}
\end{array}\right]^{(k)}\left\{\begin{array}{c}
\frac{\partial u}{\partial x}+\sum_{I=1}^{N} \frac{\partial U^{I}}{\partial x} \Phi^{I} \\
\frac{\partial v}{\partial y}+\sum_{I=1}^{N} \frac{\partial V^{I}}{\partial y} \Phi^{I} \\
\gamma_{x y}=\frac{\partial u}{\partial y}+\frac{\partial v}{\partial x}+\sum_{I=1}^{N}\left(\frac{\partial U^{I}}{\partial y}+\frac{\partial V^{I}}{\partial x}\right) \Phi^{I}
\end{array}\right\}
$$



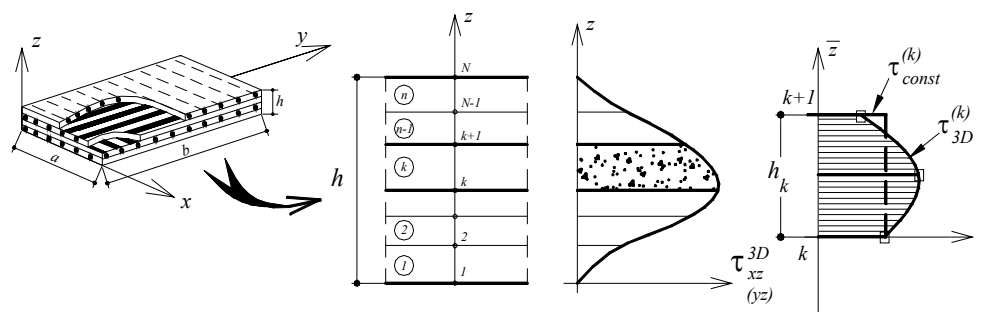

Fig. 9. Shear stresses satisfying $3 \mathrm{D}$ equilibrium equations

Shear stresses can be computed by assuming quadratic variation of shear stresses within each layer (Fig. 8). This require $3 n$ equations for each of shear stresses $\left(\tau_{x z}^{(k)}, \tau_{y z}^{(k)}\right)$, where $n$ is the number of layers. These equations can be obtained from the following conditions:

(1) satisfying traction free boundary conditions at the bottom and top surface of the plate (2 equations):

$$
\tau^{(1)}(\bar{z}=0)=\tau^{(n)}\left(\bar{z}=h_{n}\right)
$$

(2) providing the continuity of stresses along interfaces ( $n-1$ equations):

$$
\tau^{(k-1)}\left(\bar{z}=h_{k-1}\right)=\tau^{(k)}(\bar{z}=0),
$$

(3) assuming the average shear stresses from the constitutive equations ( $n$ equations) :

$$
\frac{1}{h_{k}} \int_{0}^{h_{k}} \tau^{(k)}(\bar{z}) d \bar{z}=\tau_{\text {const. }}^{(k)}
$$

(4) and computing the jump in $\left(\tau_{x z, z}^{(k)} \tau_{y z, z}^{(k)}\right)$ at each interface utilizing the first two equations of equilibrium in terms of stresses ( $n-1$ equations):

$$
\frac{\partial \tau^{(k-1)}\left(\bar{z}=h_{k-1}\right)}{\partial \bar{z}}-\frac{\partial \tau^{(k)}(\bar{z}=0)}{\partial \bar{z}}=\frac{\partial \tau_{3 D}^{(k-1)}}{\partial z}-\frac{\partial \tau_{3 D}^{(k)}}{\partial z}
$$

\section{NUMERICAL EXAMPLES}

We considered cross-ply laminated plate, in which each lamina is made of material:

$$
E_{1} / E_{2}=25, \quad G_{12} / E_{2}=G_{13} / E_{2}=0.5, \quad G_{23} / E_{2}=0.2, \quad v_{12}=v_{13}=v_{23}=0.25
$$

The results are presented in the following normalized form:

$$
\bar{w}=\frac{E_{2} 100 h^{3}}{q_{0} a^{4}} w,\left(\bar{\sigma}_{x x}, \bar{\sigma}_{y y}, \bar{\tau}_{x y}\right)=\left(\frac{h}{a}\right)^{2} \frac{1}{q_{0}}\left(\sigma_{x x}, \sigma_{y y}, \tau_{x y}\right),\left(\bar{\tau}_{x z}, \bar{\tau}_{y z}\right)=\left(\frac{h}{a}\right) \frac{1}{q_{0}}\left(\tau_{x z}, \tau_{y z}\right)
$$

where $h$ is plate thickness, $a, b$ are plate dimensions in $x, y$ directions, respectively. 
The transverse deflection and stresses are computed at the following locations:

$$
\begin{aligned}
& \bar{w}=\bar{w}\left(\frac{a}{2}, \frac{b}{2}, 0\right), \bar{\sigma}_{x x}=\bar{\sigma}_{x x}\left(\frac{a}{2}, \frac{b}{2}, \pm \frac{h}{2}\right), \bar{\sigma}_{y y}=\bar{\sigma}_{y y}\left(\frac{a}{2}, \frac{b}{2}, \pm \frac{h}{4}\right), \bar{\tau}_{x y}=\bar{\sigma}_{x x}\left(0,0, \pm \frac{h}{2}\right) \\
& \bar{\tau}_{x z}=\bar{\tau}_{x z}\left(\frac{a}{2}, 0,0\right), \quad \bar{\tau}_{y z}=\bar{\tau}_{y z}\left(0, \frac{b}{2}, 0\right)
\end{aligned}
$$

Results are obtained using program $\boldsymbol{C S} \_\boldsymbol{G L P T}$ coded in MATLAB.

4.1 Example I: Cross-ply plate loaded by bi-harmonic distribution of transverse pressure A cross-ply plate is loaded by bi-harmonic distribution of transverse pressure:

$$
q(x, y)=q_{0} \sin \frac{\pi}{a} x \sin \frac{\pi}{b} y
$$

shown on Fig. 10.

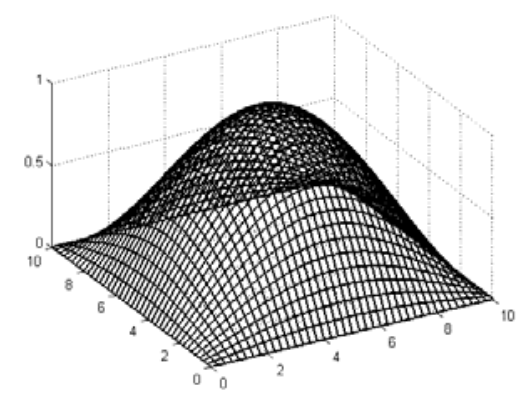

Fig. 10. Example I: bi-harmonic distribution of transverse pressure

Square, symmetrically laminated plate made of nine layers $0^{0} / 90^{\circ} / 0^{0} / 90^{\circ} / 0^{0} / 90^{0} / 0^{0} / 90^{\circ} / 0^{0}$ is analyzed. Results are shown in Table 1. and Fig. 11.

Table 1. Example I: Comparison between 3D and GLPT

\begin{tabular}{|c|c|c|c|c|c|c|c|}
\hline a/h & Solution & $\bar{w}$ & $\bar{\sigma}_{x x}$ & $\bar{\sigma}_{y y}$ & $\bar{\tau}_{x y}$ & $\bar{\tau}_{x z}$ & $\bar{\tau}_{y z}$ \\
\hline \multirow{3}{*}{4} & $\mathbf{3 Д}^{[1]}$ & - & 0.684 & 0.628 & 0.0337 & 0.2134 & 0.223 \\
\cline { 2 - 8 } & GLPT $^{[2]}$ & $\begin{array}{c}1.797 \\
1\end{array}$ & $\begin{array}{c}0.671 \\
8\end{array}$ & $\begin{array}{c}0.618 \\
7\end{array}$ & $\begin{array}{c}0.0346 \\
6\end{array}$ & 0.223 & $\begin{array}{c}0.232 \\
5\end{array}$ \\
\hline \multirow{3}{*}{$\mathbf{1 0}$} & $\mathbf{3 Д}^{[1]}$ & - & 0.551 & 0.477 & 0.0235 & 0.247 & 0.226 \\
\cline { 2 - 8 } & GLPT $^{[2]}$ & $\begin{array}{c}0.657 \\
6\end{array}$ & $\begin{array}{c}0.552 \\
1\end{array}$ & $\begin{array}{c}0.471 \\
7\end{array}$ & $\begin{array}{c}0.0237 \\
5\end{array}$ & $\begin{array}{c}0.2118 \\
1\end{array}$ & $\begin{array}{l}0.261 \\
1\end{array}$ \\
\hline \multirow{2}{*}{$\mathbf{1 0}$} & $\mathbf{3}^{[1]}$ & - & 0.539 & 0.431 & 0.0213 & 0.219 & 0.259 \\
\cline { 2 - 8 } & GLPT $^{[2]}$ & $\begin{array}{c}0.433 \\
5\end{array}$ & $\begin{array}{c}0.538 \\
8\end{array}$ & $\begin{array}{c}0.419 \\
6\end{array}$ & $\begin{array}{c}0.0213 \\
1\end{array}$ & 0.2015 & $\begin{array}{c}0.275 \\
9\end{array}$ \\
\hline
\end{tabular}



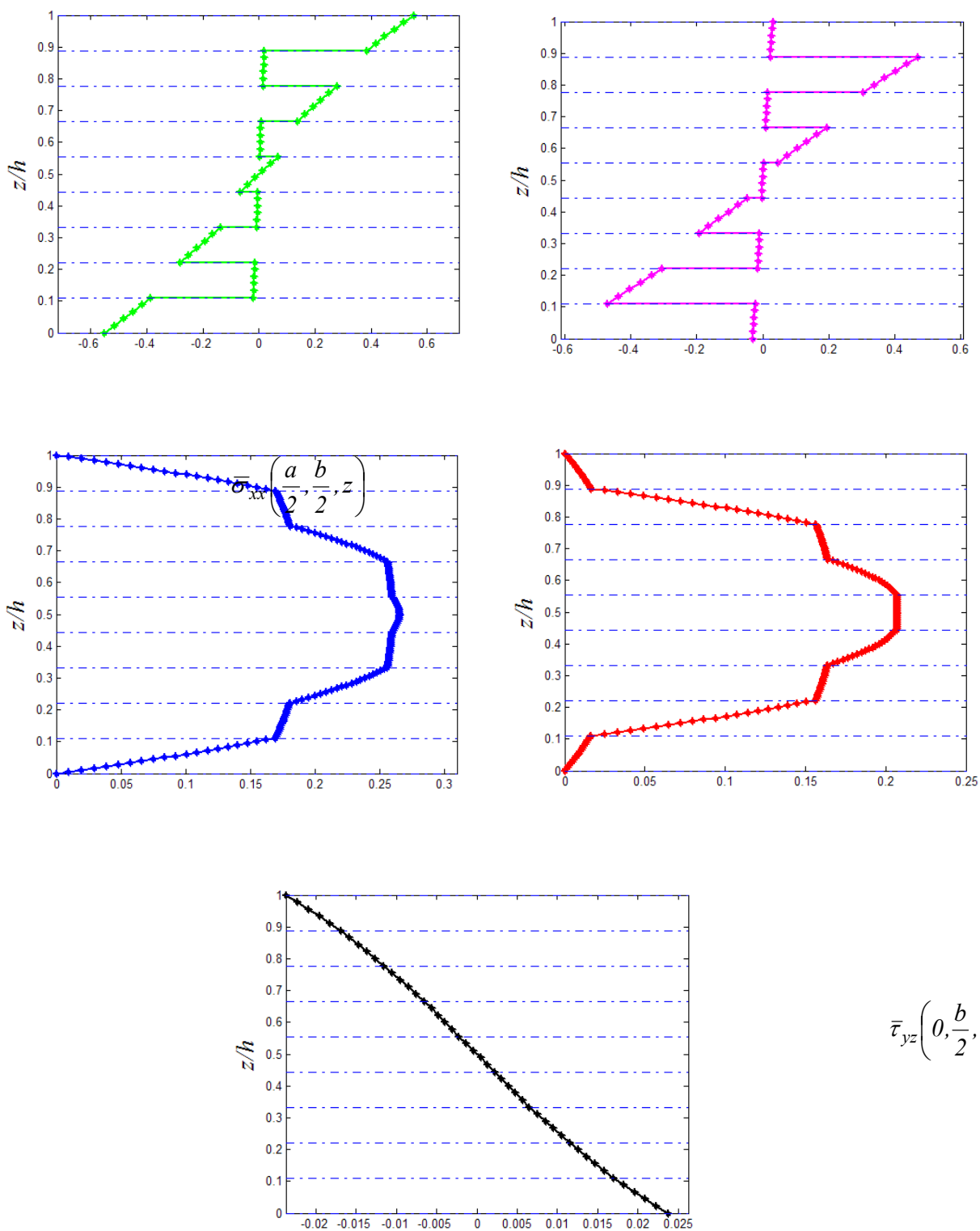

$$
\bar{\tau}_{y z}\left(0, \frac{b}{2},\right.
$$

Fig. 11. Normalized stresses for square plate $(a / h=10)$ made of nine layers, loaded with bi-harmonic transverse pressure

4.2 Example II: Cross-ply plate loaded by transverse distribution of constant pressure A cross-ply plate is loaded transverse distribution of constant pressure:

$$
q(x, y)=q_{0}
$$

shown on Fig. 12. 


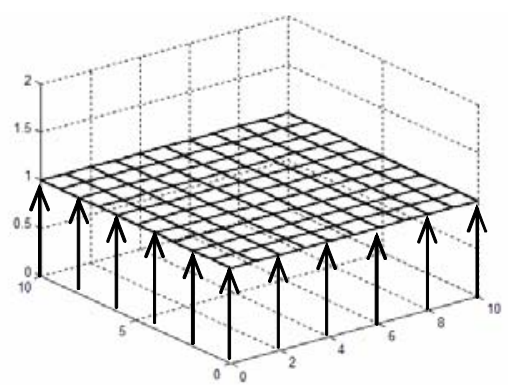

Fig. 12. Example II: transverse distribution of constant pressure

Square, symmetrically laminated plate made of three layers $0^{0} / 90^{\circ} / 0^{0}$ is analyzed. Results are shown in Table 2.

Table 2. Example II: Comparison between GLPT and 3D, 2D

\begin{tabular}{|c|c|c|c|c|c|c|c|}
\hline$a / h$ & $\begin{array}{c}\text { Solution } \\
s\end{array}$ & $\bar{w}$ & $\bar{\sigma}_{x x}$ & $\bar{\sigma}_{y y}$ & $\bar{\tau}_{x y}$ & $\bar{\tau}_{x z}$ & $\bar{\tau}_{y z}$ \\
\hline \multirow{4}{*}{4} & GLPT $^{[2]}$ & $\begin{array}{c}3.079 \\
0\end{array}$ & $\begin{array}{c}1.115 \\
7\end{array}$ & $\begin{array}{c}0.780 \\
4\end{array}$ & 0.1094 & 0.4552 & 0.5214 \\
\hline & $C C^{[3]}$ & $\begin{array}{c}3.044 \\
4\end{array}$ & $\begin{array}{c}1.117 \\
3\end{array}$ & - & $\begin{array}{c}0.0973 \\
4\end{array}$ & 0.4435 & 0.4956 \\
\hline & FSDT & $\begin{array}{c}2.334 \\
4\end{array}$ & $\begin{array}{c}0.667 \\
6\end{array}$ & - & $\begin{array}{c}0.0692 \\
3\end{array}$ & $\begin{array}{c}0.6466 \\
9\end{array}$ & $\begin{array}{c}0.5312 \\
5\end{array}$ \\
\hline & CLPT & $\begin{array}{c}0.658 \\
8\end{array}$ & $\begin{array}{c}0.804 \\
0\end{array}$ & - & $\begin{array}{c}0.0418 \\
9\end{array}$ & $\begin{array}{c}0.7211 \\
6\end{array}$ & $\begin{array}{c}0.3842 \\
6\end{array}$ \\
\hline \multirow{4}{*}{10} & GLPT $^{[2]}$ & $\begin{array}{c}1.156 \\
4 \\
\end{array}$ & $\begin{array}{c}0.872 \\
1 \\
\end{array}$ & $\begin{array}{c}0.359 \\
7 \\
\end{array}$ & $\begin{array}{c}0.0611 \\
7 \\
\end{array}$ & 0.6305 & 0.4087 \\
\hline & $\mathrm{CC}^{[3]}$ & $\begin{array}{c}1.154 \\
1 \\
\end{array}$ & $\begin{array}{c}0.870 \\
8 \\
\end{array}$ & - & $\begin{array}{c}0.0597 \\
4 \\
\end{array}$ & 0.6279 & 0.4017 \\
\hline & FSDT & $\begin{array}{c}0.954 \\
6\end{array}$ & $\begin{array}{c}0.773 \\
3\end{array}$ & - & $\begin{array}{c}0.0494 \\
3\end{array}$ & $\begin{array}{c}0.7060 \\
0\end{array}$ & $\begin{array}{l}0.4150 \\
7\end{array}$ \\
\hline & CLPT & $\begin{array}{c}0.658 \\
8 \\
\end{array}$ & $\begin{array}{c}0.804 \\
0 \\
\end{array}$ & - & $\begin{array}{c}0.0418 \\
9 \\
\end{array}$ & $\begin{array}{c}0.7211 \\
6\end{array}$ & $\begin{array}{l}0.3842 \\
6 \\
\end{array}$ \\
\hline \multirow{4}{*}{$\begin{array}{c}10 \\
0\end{array}$} & GLPT $^{[2]}$ & $\begin{array}{c}0.671 \\
3\end{array}$ & $\begin{array}{c}0.808 \\
3\end{array}$ & $\begin{array}{c}0.193 \\
5\end{array}$ & $\begin{array}{c}0.0428 \\
5 \\
\end{array}$ & 0.7201 & 0.3854 \\
\hline & $\mathrm{CC}^{[3]}$ & $\begin{array}{c}0.671 \\
3\end{array}$ & $\begin{array}{c}0.808 \\
3\end{array}$ & - & $\begin{array}{c}0.0428 \\
5\end{array}$ & 0.7201 & 0.3852 \\
\hline & FSDT & $\begin{array}{c}0.661 \\
8 \\
\end{array}$ & $\begin{array}{c}0.803 \\
7\end{array}$ & - & $\begin{array}{c}0.0420 \\
2 \\
\end{array}$ & $\begin{array}{c}0.7209 \\
6\end{array}$ & $\begin{array}{c}0.3845 \\
7 \\
\end{array}$ \\
\hline & CLPT & $\begin{array}{c}0.658 \\
8 \\
\end{array}$ & $\begin{array}{c}0.804 \\
0 \\
\end{array}$ & - & $\begin{array}{c}0.0418 \\
9 \\
\end{array}$ & $\begin{array}{c}0.7211 \\
6 \\
\end{array}$ & $\begin{array}{c}0.3842 \\
6 \\
\end{array}$ \\
\hline
\end{tabular}

${ }^{[1]}$ Pagano N.J.

${ }^{[2]}$ CS_GLPT - MATLAB program

${ }^{[3]}$ E. Carrera, A. Ciuffreda

CLPT - Classical Laminate Plate Theory

FSDT - First-order Shear Deformation Theory 


\section{CONCLUSION}

The proposed mathematical model can be used for analysis of thick, as well as thin plates. It is shown that differences among $2 \mathrm{D}$ and $3 \mathrm{D}$ theories are greater for transverse shear stresses, than for in-plane stresses. Also, differences among theories vanish by increasing $a / h$. Finally, as GLPT model is capable to achieve the same solution accuracy as conventional 3D elasticity model, it can be used as test model of approximation models.

\section{REFERENCES}

1. Reddy, J. N., Mechanics Of Laminated Composite Plates, CRC Press, 1996

2. Reddy, J. N., A plate bending element based on a generalized laminated plate theory, International Journal For Numerical Methods In Engineering, vol. 28, 2275-2292, 1989

3. Chaudhuri, R. A., An Approximate Semi-Analytical Method For Prediction Of Interlaminar Shear Stress In Arbitrarily Laminated Thick Plate, Comp. Struct., 25, 627-636, 1987

4. Carrera, E., Ciuffreda, A., A Unified Formulation To Asses Theories Of Multilayered Plates For Various Bending Problems, Composite Structures, in press.

\section{ANALITIČKO REŠENJE VIŠESLOJNIH PLOČA ZASNOVANO NA OPŠTOJ LAMINATNOJ TEORIJI PLOČA}

\section{Đorđe Vuksanović, Marina Ćetković}

U ovom radu prikazano je anlitičko rešenje statičke anlize slobodno oslonjene kompzitne ploče, zasnovano na opštoj laminatnij teoriji ploča (GLPT). Matematički model pretpostavlja deo po deo linearnu raspodelu komponenata pomeranja $u_{1}, u_{2} i$ konstantnu promenu komponente pomeranja $u_{3}$ po debljini ploče. Pomenuti model u obzir uzima i uticaj smičuće deforamcije unutar svakog sloja, čime se dobija realna procena smicanja po debljini ploče. Naime, smičući naponi zadovoljavaju Hook-ov zakon, 3D uslove ravnoteže i granične uslove po naponima. Koristeći pretpostavljeno polje pomeranja, linearne veze deformacija i pomeranja i konstitutivne jednačine lamine, primenom principa virtualnih pomeranja izvedeni su uslovi ravnoteže. Navier-ovo rešenje GLPT prikazano je za slobodno oslonjenu ploču, sačinjenu od ortotropnih lamina, opterećenu biharmonijskom i konstantnom raspodelom poprečnog opterećenja. Dobijeno je izvrsno slaganje sa $3 D$ rešenjem elastične teorije. 